ARTICLE

Received 2 May 2014 | Accepted 17 Nov 2014 | Published 6 Jan $2015 \quad$ DOl: 10.1038/ncomms6881

\title{
Sub-10 nm rutile titanium dioxide nanoparticles for efficient visible-light-driven photocatalytic hydrogen production
}

\author{
Landong $\mathrm{Li}^{1,2, \star}$, Junqing Yan ${ }^{1,2, \star}$, Tuo Wang ${ }^{1,3}$, Zhi-Jian Zhao ${ }^{1,3}$, Jian Zhang ${ }^{4}$, Jinlong Gong ${ }^{1,3}$ \& Naijia Guan ${ }^{1,2}$
}

Titanium dioxide is a promising photocatalyst for water splitting, but it suffers from low visible light activity due to its wide band gap. Doping can narrow the band gap of titanium dioxide; however, new charge-carrier recombination centres may be introduced. Here we report the design of sub-10 nm rutile titanium dioxide nanoparticles, with an increased amount of surface/sub-surface defects to overcome the negative effects from bulk defects. Abundant defects can not only shift the top of the valence band of rutile titanium dioxide upwards for band-gap narrowing but also promote charge-carrier separation. The role of titanium(III) is to enhance, rather than initiate, the visible-light-driven water splitting. The sub-10 nm rutile nanoparticles exhibit the state-of-the-art activity among titanium dioxide-based semiconductors for visible-light-driven water splitting and the concept of ultra-small nanoparticles with abundant defects may be extended to the design of other robust semiconductor photocatalysts.

\footnotetext{
${ }^{1}$ Collaborative Innovation Center of Chemical Science and Engineering, Tianjin 300072, China. ${ }^{2}$ Key Laboratory of Advanced Energy Materials Chemistry of Ministry of Education, College of Chemistry, Nankai University, Tianjin 300071, China. ${ }^{3}$ Key Laboratory for Green Chemical Technology of Ministry of Education, School of Chemical Engineering and Technology, Tianjin University, 92 Weijin Road, Nankai District, Tianjin 300072, China. ${ }^{4}$ Department of New Energy Technology, Ningbo Institute of Materials Technology and Engineering, Chinese Academy of Sciences, Tianjin, Ningbo 315201, China. * These authors contributed equally to this work. Correspondence and requests for materials should be addressed to J.G. (email: jlgong@tju.edu.cn).
} 
S ince the discovery of water photolysis on a $\mathrm{TiO}_{2}$ photoanode in the $1970 \mathrm{~s}^{1}$, semiconductor photocatalysis has attracted significant attention due to its promising applications in environment remediation and solar energy conversion in the past decades ${ }^{2-5} . \mathrm{TiO}_{2}$ is the initial semiconductor photocatalyst investigated and it is still regarded as a benchmark photocatalyst under ultravoilet irradiation due to its intrinsic high activity. However, $\mathrm{TiO}_{2}$ is a type of wide bandgap semiconductor and it only adsorbs ultravoilet light, which greatly limits its practical applications ${ }^{6}$. Accordingly, persistent efforts have been made to narrow the band gap of $\mathrm{TiO}_{2}$ to extend its working spectrum to the visible light region, that is, so-called band-gap engineering.

Doping with metal or non-metal elements is known as a feasible means to tune the electronic structure of $\mathrm{TiO}_{2}$ and to introduce new states into the $\mathrm{TiO}_{2}$ band gap for visible light response $^{7-17}$. In general, doping with non-metal elements, for example, $\mathrm{C}, \mathrm{N}$ and $\mathrm{S}$, can build acceptor states above the valence band from the $p$ states of non-metal ions ${ }^{16}$, and doping with metal elements, for example, $\mathrm{Fe}$ and $\mathrm{Cr}$, can build donor states below the conduction band ${ }^{2}$. For doped $\mathrm{TiO}_{2}$, the lattice defects induced by the dopants will unavoidably introduce new chargecarrier trapping and recombination centres, which might correspondingly show a degrading effect on the photocatalytic activity ${ }^{7}$. Moreover, doped $\mathrm{TiO}_{2}$ materials are usually not adequate catalysts for photocatalytic hydrogen production from water splitting, although their superior activity in photocatalytic oxidation reactions have been well documented ${ }^{6,18}$. Self-doping with $\mathrm{Ti}^{3+}$ was further developed for narrowing the band of $\mathrm{TiO}_{2}$ without the introduction of unwanted carrier recombination centres from dopants ${ }^{19-21}$, which consequently exhibited good stability and considerable activity for photocatalytic hydrogen production under visible light ${ }^{20,21}$. Recently, hydrogenation of crystalline $\mathrm{TiO}_{2}$ was disclosed as a new approach to enhance the visible as well as infrared light absorption of pristine $\mathrm{TiO}_{2}$ and subsequently triggered great interest ${ }^{22-28}$. The surface disorder of hydrogenated black $\mathrm{TiO}_{2}$, instead of bulk $\mathrm{Ti}^{3+}$, was proposed to be responsible for the extended light absorption ${ }^{23,24}$. The hydrogenated $\mathrm{TiO}_{2}$ exhibited remarkable activity in the photocatalytic hydrogen production from water splitting under full solar irradiation. However, considering that a sharp decline in the hydrogen production rate by two orders of magnitude (for example, from 10 to $\left.0.1 \mathrm{mmol} \mathrm{h}^{-1} \mathrm{~g}^{-1}\right)^{22,25}$ was observed if ultraviolet light was filtered out $(\lambda>400 \mathrm{~nm})$, the original hydrogen production activity should more probably come from the enhanced charge separation and transportation under ultraviolet irradiation rather than the extended visible light absorption $^{29}$.

The possibility of extending the working spectrum of $\mathrm{TiO}_{2}$ to the visible light region is generally acknowledged based on both experimental observations and theoretical calculations ${ }^{7-32}$. The common point of different strategies lies in disrupting the integrity of ordered lattice structure of pristine $\mathrm{TiO}_{2}$ (rutile or anatase) and building new states within the band gap for photoexcitation with lower energy. However, despite recent achievements, a simple strategy to $\mathrm{TiO}_{2}$-based semiconductors for efficient visible-light-driven photocatalytic hydrogen production is still challenging. Indeed, the successful photocatalyst system should fulfill all the requirements simultaneously: (i) narrowed band gap for visible light response; (ii) delicately designed band edge positions to realize photocatalytic redox reaction; and (iii) high efficiency for chargecarrier separation to promote photocatalytic activity.

Here we present a direct hydrolysis route to sub-10 $\mathrm{nm}$ rutile $\mathrm{TiO}_{2}$ nanoparticles for efficient photocatalytic hydrogen production under visible light irradiation. The simple strategy leads to state-of-the-art photocatalytic activity among $\mathrm{TiO}_{2}$-based semiconductors, and the simplified rutile $\mathrm{TiO}_{2}$ semiconductor system can provide information on the essence of defect-induced visible light photocatalytic activity.

\section{Results}

Preparation and characterization of rutile $\mathrm{TiO}_{2}$ samples. Anatase and rutile $\mathrm{TiO}_{2}$, both with tetragonal structure, are commonly used in photocatalytic reactions. Rutile $\mathrm{TiO}_{2}$ has a band gap ca. $0.2 \mathrm{eV}$ lower than that of anatase (3.0 versus $3.2 \mathrm{eV}$ ), and this could be crucial to the band-gap narrowing to extend its working spectrum to the visible light region. Bulk rutile $\mathrm{TiO}_{2}$ can be obtained via the high temperature calcination of anatase $\mathrm{TiO}_{2}$ at temperatures higher than $773 \mathrm{~K}$, while rutile $\mathrm{TiO}_{2}$ nanostructures can be prepared via a hydrothermal route or a $^{33,34}$ or direct hydrolysis route ${ }^{35-37}$. In the present work, the direct hydrolysis of $\mathrm{TiCl}_{4}$, free of any additives, is employed to prepare rutile $\mathrm{TiO}_{2}$ nanoparticles.

Experimentally, the hydrolysis of $\mathrm{TiCl}_{4}$ solution $\left(\mathrm{TiCl}_{4} /\right.$ $\mathrm{H}_{2} \mathrm{O}=1: 3 \mathrm{v} / \mathrm{v}$ ) can produce rutile $\mathrm{TiO}_{2}$ nanoparticles below $10 \mathrm{~nm}$. We need to emphasize that the rapid hydrolysis and evaporation not only ensure the formation of rutile $\mathrm{TiO}_{2}$ nanoparticles instead of nanorods in the presence of concentrated chloride ions but also create abundant defects in the as-obtained rutile $\mathrm{TiO}_{2}$ nanoparticles (vide infra).

As shown in Fig. 1a, the sample prepared via hydrolysis route followed by calcination at $473 \mathrm{~K}, \mathrm{~T}-1$, and the reference sample prepared via hydrothermal route followed by calcination at $473 \mathrm{~K}, \mathrm{~T}-3$, give the typical diffraction patterns of rutile $\mathrm{TiO}_{2}$ (JCPDF\#21-1276), indicating that pure rutile phase is obtained. The crystallinity of $\mathrm{T}-1$ is relatively low and the crystallite size is calculated to be $c a$. $9 \mathrm{~nm}$ according to the Scherrer equation from the broadening of rutile (110) reflection. Calcination at $673 \mathrm{~K} \mathrm{can}$, on the one hand, greatly enhance the crystallinity of rutile $\mathrm{TiO}_{2}$ but result in a significant increase in the crystallite size of T-2 (Table 1). Raman spectra of the samples with relative high crystallinity, that is, T-2 and T-3, show three Raman-active modes of multi-proton process $\left(230 \mathrm{~cm}^{-1}\right), E_{\mathrm{g}}\left(440 \mathrm{~cm}^{-1}\right)$ and $A_{1 \mathrm{~g}}\left(610 \mathrm{~cm}^{-1}\right)$, corresponding to the tetragonal space group of $P 42 / \mathrm{mnm}^{38}$. For T-1, an obvious blue shift and broadening of the $E_{\mathrm{g}}$ band $\left(\sim 25 \mathrm{~cm}^{-1}\right.$, Fig. 1b) is clearly observed due to the photon-confinement effects induced by defects ${ }^{23,39}$.

X-ray photoelectron spectroscopy (XPS) analysis is performed to study the surface and sub-surface chemical states (in the depth up to $3 \mathrm{~nm}$ ) of rutile $\mathrm{TiO}_{2}$ samples. In Ti $2 p$ spectra (Fig. 1c), two peaks at binding energy of 458.6 and $464.1 \mathrm{eV}$, assignable to $2 p_{3 / 2}$ and $2 p_{1 / 2}$, respectively, of $\mathrm{Ti}^{4}+$ in $\mathrm{TiO}_{2}$ (ref. 40 ), are observed for all rutile $\mathrm{TiO}_{2}$ samples, indicating the identical chemical state of $\mathrm{Ti}$ atoms in these samples. No obvious $\mathrm{Ti}^{3+}$ signals could be observed in Ti $2 p$ spectra. In O 1-s spectra (Fig. 1d), a wellformed peak at $529.6 \mathrm{eV}$ and two shoulders at 531.7 and $533.1 \mathrm{eV}$ are observed for samples prepared by hydrolysis. The binding energy value of $529.6 \mathrm{eV}$ is attributed to the lattice oxygen in $\mathrm{TiO}_{2}$ (ref. 23), while the binding energy values of 531.7 and $533.1 \mathrm{eV}$ are attributed to bridging hydroxyls and physisorbed water, respectively ${ }^{41,42}$. Hydrothermal synthesized sample T-3 exhibits a week peak shape at $531.7 \mathrm{eV}$ corresponding to bridging hydroxyl groups, revealing its fine crystal structure with few surface defects. The presence of bridging surface hydroxyls in rutile $\mathrm{TiO}_{2}$ samples is further supported by Fourier transform infrared spectroscopy (FTIR) with the observation of strong broad band located at $3,425 \mathrm{~cm}^{-1}$ (Supplementary Fig. 1). It is interesting to note that the percentages of hydroxyls in total oxygen species for $\mathrm{T}-1$ is slightly higher than that of T-2, both much higher than that of 

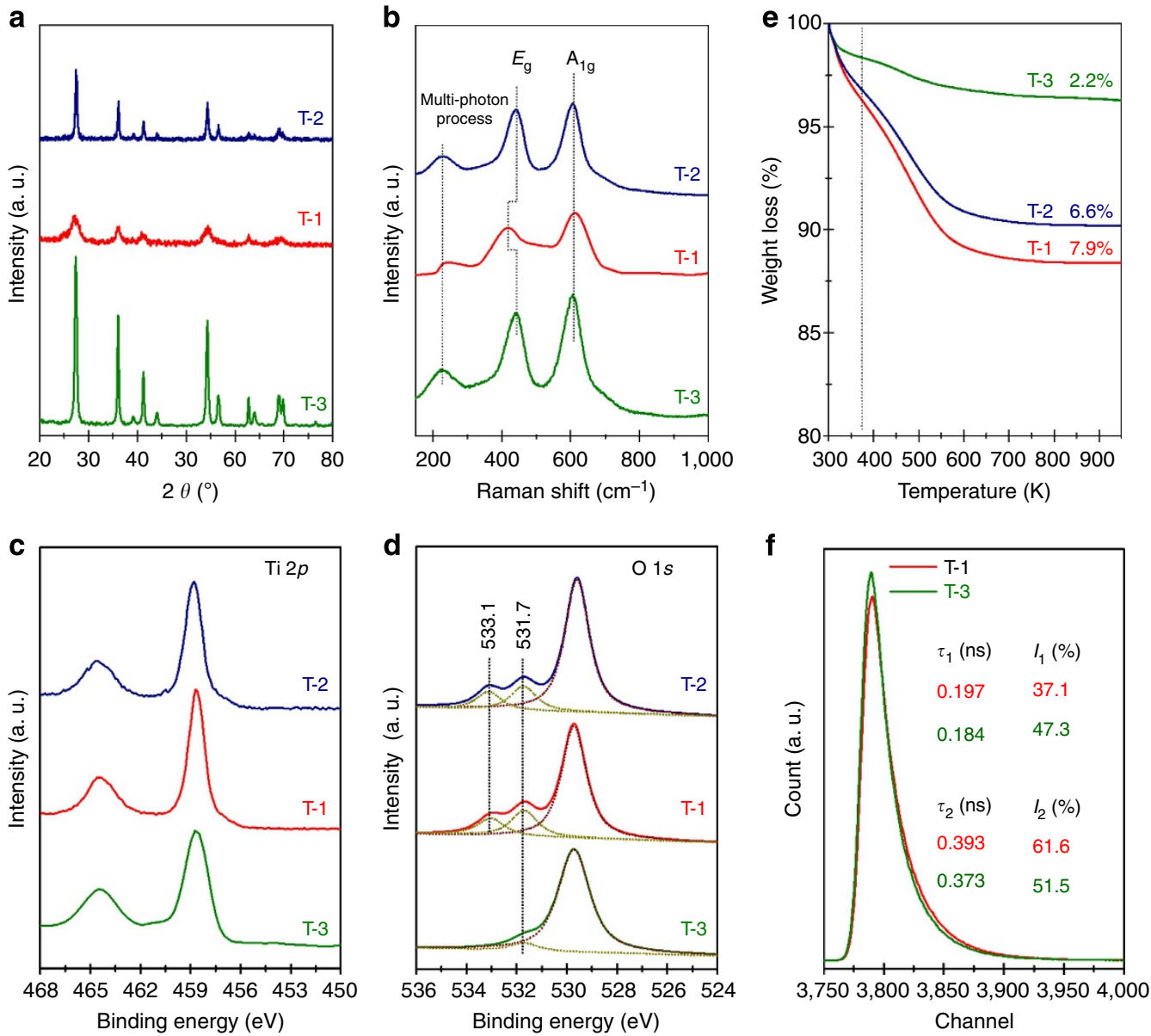

Figure 1 | Spectroscopy charatcerization of rutile $\mathrm{TiO}_{\mathbf{2}}$ samples. (a) XRD patterns of rutile $\mathrm{TiO}_{2}$ samples. (b) Raman spectra of rutile $\mathrm{TiO}_{2}$ samples. (c,d) $\mathrm{Ti} 2 p$ and $\mathrm{O} 1 \mathrm{~s}$ XPS of rutile $\mathrm{TiO}_{2}$ samples. (e) Thermogravimetry analysis of rutile $\mathrm{TiO}_{2}$ samples. (f) Positron annihilation lifetime spectra of rutile $\mathrm{TiO}_{2}$ samples with corresponding positron lifetime and relative intensity in the inset.

Table 1 | Physico-chemical properties of $\mathrm{TiO}_{2}$ samples under study.

\begin{tabular}{|c|c|c|c|c|c|c|c|c|}
\hline$\frac{\text { Sample }}{T-1}$ & $\begin{array}{l}\text { Preparation strategy } \\
\text { Hydrolysis at } 373 \mathrm{~K} \\
\text { Calcined at } 473 \mathrm{~K}\end{array}$ & $\begin{array}{l}\text { Crystalline phase } \\
\text { Rutile }\end{array}$ & \multicolumn{2}{|c|}{ Crystallite size (nm) } & $\frac{\text { BET }\left(\mathbf{m}^{\mathbf{2}} \mathbf{g}^{-\mathbf{1}}\right)}{101}$ & \multicolumn{2}{|c|}{ OH groups (\%) } & $\frac{\text { Color }}{\text { Light grey }}$ \\
\hline $\mathrm{T}-2$ & $\begin{array}{l}\text { Hydrolysis at } 373 \mathrm{~K} \\
\text { Calcined at } 673 \mathrm{~K}\end{array}$ & Rutile & $32^{\star}$ & $27^{\dagger}$ & 86 & $14.3^{\ddagger}$ & $15.9^{\S}$ & White \\
\hline T-3 & $\begin{array}{l}\text { Hydrothermal at } 453 \mathrm{~K} \\
\text { Calcined at } 473 \mathrm{~K}\end{array}$ & Rutile & $23^{\star}$ & $16 \times 70^{\dagger}$ & 55 & $4.9^{\ddagger}$ & $5.2^{\S}$ & White \\
\hline $\mathrm{T}-4$ & $\begin{array}{l}\text { Hydrothermal at } 373 \mathrm{~K} \\
\text { Calcined at } 473 \mathrm{~K}\end{array}$ & Anatase & $6^{\star}$ & $7^{\dagger}$ & 208 & / & I & White \\
\hline $\begin{array}{l}\text { TEM, trans } \\
{ }^{*} \text { Calculated } \\
\text { †TEM obse } \\
\text { †Calculated } \\
\text { \$Calculated }\end{array}$ & $\begin{array}{l}\text { on electron microscopy; TG, ther } \\
\text { cherrer equation from XRD patte } \\
\text { ns. } \\
\text { O 1-s XP spectra. } \\
\text { TG analysis in the temperature }\end{array}$ & $\begin{array}{l}\text { imetry; XP, X-ray ph } \\
\text { of } 373-973 \mathrm{~K} \text {. }\end{array}$ & & & & & & \\
\hline
\end{tabular}

hydrothermal-synthesized T-3 (Table 1). This is further confirmed by the thermogravimetry analysis in Fig. 1e, where weight losses of $7.9 \%$ and $2.2 \%$ in the temperature range of $373-$ $973 \mathrm{~K}$ are observed for T-1 and T-3, respectively.

Positron annihilation is a well-established technique to study the defects in semiconductor materials and the lifetime of the positron is able to give information on the nature of various defects down to the p.p.m. level ${ }^{43}$. We have employed this technique to investigate the defects in rutile $\mathrm{TiO}_{2}$ samples prepared via direct hydrolysis and hydrothermal synthesis, and the results are shown in Fig. 1f. Two different fitted curves are clearly observed and the corresponding fitting parameters, lifetime, $\tau_{1}, \tau_{2}$ and $\tau_{3}$, with relative intensity, $I_{1}, I_{2}$ and $I_{3}$, are summarized in the inset. The longest lifetime component $\tau_{3}$ is attributed to the annihilation of orthopositronium atoms formed in very large voids presented in the material ${ }^{44}$. The lifetime components $\tau_{1}$ and $\tau_{2}$ have been demonstrated to indicate the existence of point defects in the bulk phase and the defects 
located on the surface of the samples, respectively ${ }^{45,46}$. The lifetime $\tau_{1}$ and $\tau_{2}$ of $\mathrm{T}-1$ is comparable with that of T-3, which indicates the similar nature of surface and bulk defects in both samples, as the nature of defects can influence the local electron density and subsequently influence the positron lifetime ${ }^{47}$. The value of $I_{2} / I_{1}$, reflecting the intensity ratio of surface to bulk defects, is calculated to be 1.66 for T-1, obviously higher than the 1.09 of $\mathrm{T}-3$, indicating the rich of surface defects in sub- $10 \mathrm{~nm}$ rutile $\mathrm{TiO}_{2}$ nanoparticles prepared via hydrolysis.

The morphology and structure of rutile $\mathrm{TiO}_{2}$ samples are analysed by transmission electron microscopy (TEM), as shown in Fig. 2a. T-1 appears as aggregates of nanoparticles with an average diameter of $c a .8 \mathrm{~nm}$. Calcination at $673 \mathrm{~K}$ results in a distinct increase in the crystallite size of rutile $\mathrm{TiO}_{2}$ from 8 to $27 \mathrm{~nm}$, basically in agreement with X-ray diffraction results. The reference sample T-3 appears as nanorods with an average diameter of $16 \mathrm{~nm}$ and a length of $70 \mathrm{~nm}$. For rutile $\mathrm{TiO}_{2},(110)$ facet is the most stable facet with lowest formation energy ${ }^{48}$ and it is observed as the dominant facet for all rutile $\mathrm{TiO}_{2}$ samples, as illustrated by the lattice fringes with a spacing of $0.325 \mathrm{~nm}$ in the high-resolution TEM images (Fig. 2b). In addition, disordered structure induced by defects, can be observed for rutile $\mathrm{TiO}_{2}$

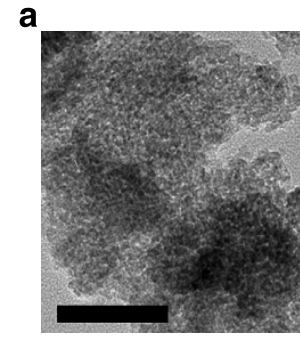

b
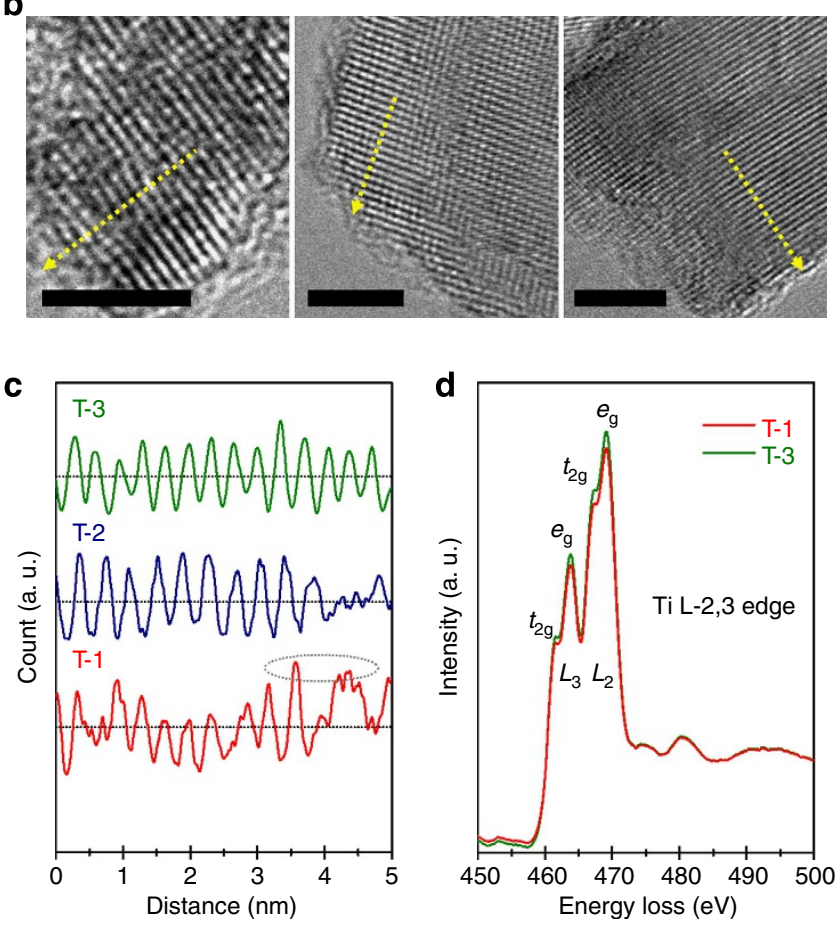

Figure 2 | Morphology and structure of rutile $\mathrm{TiO}_{2}$ samples. (a) Overview TEM images of rutile $\mathrm{TiO}_{2}$ samples. Scale bar, $50 \mathrm{~nm}$. (b) High-resolution TEM (HRTEM) images of rutile $\mathrm{TiO}_{2}$ samples. Scale bar, $4 \mathrm{~nm}$.

(c) Structural analysis based on HRTEM images, analysis region and orientation marked in the HRTEM images. (d) $\mathrm{Ti} L_{2,3}$ edge EELS of rutile $\mathrm{TiO}_{2}$ samples. samples prepared via direct hydrolysis (T-3). Structural analysis based on high-resolution TEM image is further performed to provide visualized information on the existence of defects in rutile $\mathrm{TiO}_{2}$ samples under study (Fig. 2c). For T-3, clearly resolved and well-defined lattice fringes are observed and the distance between the adjacent lattice planes is equivalent to standard spacing, revealing the high crystallinity and the formation of fine crystals. For T-1, different types of defects, including intrinsic bulk defects (different distances between adjacent lattice planes) and surface hydroxyls (higher intensity trace, as marked with circle in Fig. 2c), can be clearly observed. Calcination at $673 \mathrm{~K}$ results in an increase in the crystallinity and the corresponding decrease in the defect degree. However, defects can still be observed, especially at the edges of nanoparticles (Fig. 2c), which is consistent with XPS results. Electron energy loss spectra (EELS) is further performed on $\mathrm{T}-1$ and $\mathrm{T}-3$, to give a qualitative interpretation of electronic states, as shown in Fig. $2 d$. For Ti $L_{2,3}$ edge, the Ti $3 d$ character splits into two groups: the threefold $t_{2 \mathrm{~g}}$ and the twofold $e_{\mathrm{g}}$ orbitals, owing to the octahedral coordination with $\mathrm{O}$ atom forming $s$-type and $\pi$-type bonds ${ }^{49}$. The $t_{2 \mathrm{~g}}-e_{\mathrm{g}}$ splitting in $\mathrm{Ti}$ $L_{2,3}$ edge of T-1 and T-3 is quite similar, ruling out the existence of $\mathrm{Ti}$ with different electronic states, that is, $\mathrm{Ti}^{3+23}$. A noticeable intensity decrease of the $L_{2}$ peak of T-1 should be originated from the existence of defects ${ }^{50,51}$.

Band gap states of as-prepared rutile $\mathrm{TiO}_{2}$ samples. The optical properties of as-prepared rutile $\mathrm{TiO}_{2}$ samples are investigated by diffuse reflectance ultraviolet-visible spectroscopy and the results are shown in Fig. 3a. T-3 shows a band-edge absorption around $410 \mathrm{~nm}$, typical for rutile $\mathrm{TiO}_{2}$ with a band-gap energy of ca. $3.0 \mathrm{eV}$. In contrast, $\mathrm{T}-1$ shows obvious visible light absorption up to $600 \mathrm{~nm}$, which should be originated from the existence of abundant defects. Calcination at $673 \mathrm{~K}$ will eliminate some of the defects (as discussed in the previous section) and therefore significantly reduce the absorption in visible light region. The plots of transformed Kubelka-Munk function versus the energy of light (Fig. 3b) give band-gap energies of 2.74, 2.84 and $2.98 \mathrm{eV}$ for T-1, T-2 and T-3, respectively. Based on its light-absorption feature, the sub-10 nm rutile $\mathrm{TiO}_{2}$ nanoparticles, $\mathrm{T}-1$, should be an active photocatalyst under visible light irradiation.

To further address the relative band structure of rutile $\mathrm{TiO}_{2}$ samples under study, the flat-band potential is measured using the electrochemical method in $0.5 \mathrm{M} \mathrm{NaSO}_{4}$ solution $(\mathrm{pH}$ 6.8) and the Mott-Schottky plots are shown in Fig. 3c. The MottSchottky plots of all the rutile $\mathrm{TiO}_{2}$ samples show a positive slope, which is typical for $n$-type semiconductors. The conduction band bottom of all rutile $\mathrm{TiO}_{2}$ samples under study is quite similar (ca. $-0.10 \mathrm{eV}$ versus normal hydrogen electrode) according to the Mott-Schottky plots. Moreover, T-1 shows a relatively smaller slope than $\mathrm{T}-3$, indicating a faster charge transfer and a higher donor density ${ }^{29,52}$. The carrier densities $\left(N_{\mathrm{d}}\right)$ of the samples can be calculated from the slopes of Mott-Schottky plots according to equation (1): ${ }^{29}$

$$
N_{\mathrm{d}}=2 /\left(e_{0} \varepsilon \varepsilon_{0}\right)\left[d\left(1 / C^{2}\right) / d V\right]^{-1}
$$

where $e_{0}$ is the electron charge $\left(1.6 \times 10^{-19} \mathrm{C}\right), \varepsilon$ is the dielectric constant of rutile $\mathrm{TiO}_{2}, \varepsilon_{0}$ is the permittivity of vacuum $\left(8.86 \times 10^{-12} \mathrm{Fm}^{-1}\right)$ and $V$ is the applied bias at the electrode. The carrier densities are calculated to be $1.10 \times 10^{18}$, $0.95 \times 10^{18}$ and $0.45 \times 10^{18} \mathrm{~cm}^{-3}$ for T-1, T-2 and T-3, respectively. As the defect sites always act as the electron donors, a higher carrier density implies the existence of a larger amount of defects.

The valence band positions of rutile $\mathrm{TiO}_{2}$ samples are determined by linear extrapolation of the leading edges of valence 

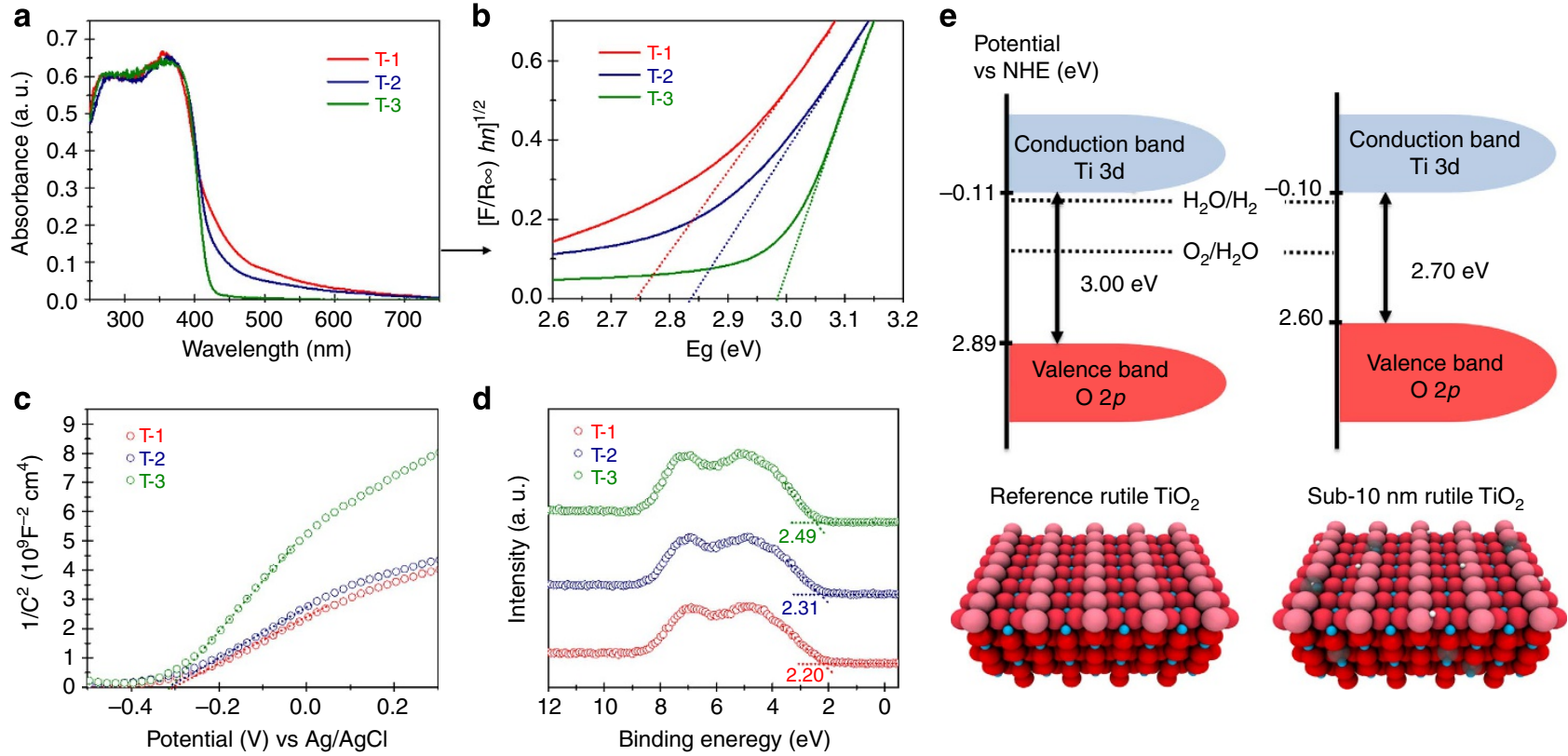

Figure 3 | Band-gap states of rutile $\mathrm{TiO}_{2}$ samples. (a,b) Diffuse reflectance ultraviolet-visible spectra of rutile $\mathrm{TiO}_{2}$ samples. (c) Mott-Schottky plots of rutile $\mathrm{TiO}_{2}$ samples. (d) VB XPS of rutile $\mathrm{TiO}_{2}$ samples. (e) Band energy diagram of reference (T-3) and sub-10 nm (T-1) rutile $\mathrm{TiO}_{2}$.

band (VB) XPS spectra to the base lines. As shown in Fig. 3d, apparent valence band values of 2.20, 2.31 and $2.49 \mathrm{eV}$ are observed for T-1, T-2 and T-3, respectively. The scatterings of data relative to the fittings are proved to be $<0.05 \mathrm{eV}$ by repeated experiments. After calibration with reference Fermi level, actual valence band values of 2.60, 2.71 and $2.89 \mathrm{eV}$ versus normal hydrogen electrode can be obtained for T-1, T-2 and T-3, respectively. Compared with $\mathrm{T}-3$, T-1 shows $c a$. $0.3 \mathrm{eV}$ upward shift in the valence band top, which is the origin of its visible absorption.

To further clarify the influence of defects on the band structure of rutile, hybrid density functional theory (DFT) calculations were done with $(2 \times 2)$ rutile $(110)$ slab models. The shifts of valence band top are summarized in Supplementary Table 1. The existence of surface defects, both bridging hydroxyls and bridging oxygen vacancy on rutile (110), indeed induces upward shift of valence band top. Moreover, higher hydroxyl coverage results in stronger shift, which agrees with the experimental observation that the valence band top of T-1, with more bridging hydroxyl, is higher in T-3. However, a qualitative comparison is not easy in this study due to the simplified model used in DFT, that is, (1) extended surface in calculation versus nanoparticle in experiment and (2) single-type defects in calculation versus coexisted multitype defects in experiment.

Based on the results from Mott-Schottky plots (Fig. 3c) and VB XPS analysis (Fig. 3d), the band gaps are calculated to be 2.70 , 2.82 and $3.00 \mathrm{eV}$ for T-1, T-2 and T-3, respectively. These values are in good accordance with the band-gap values directly measured by ultraviolet-visible spectroscopy (Supplementary Table 2). The detailed band diagrams of reference and sub$10 \mathrm{~nm}$ rutile $\mathrm{TiO}_{2}$ are depicted in Fig. 3e. Compared with reference T-3, sub-10 nm T-1 exhibits similar conduction band bottom but distinct higher valence band top. Therefore, a bandgap narrowing of $c a .0 .3 \mathrm{eV}$ could be achieved, which indicates that T-1 is a promising visible-light photoactive semiconductor. Moreover, the band-edge position reveals that the T-1 is suitable for photocatalytic hydrogen production, as its conduction band bottom is more negative than the reduction potential of $\mathrm{H}_{2} \mathrm{O} / \mathrm{H}_{2}$ and its valence band top is more positive than the oxidation potential of $\mathrm{O}_{2} / \mathrm{H}_{2} \mathrm{O}$.
Photocatalytic hydrogen production from water splitting. The photocatalytic activity of rutile $\mathrm{TiO}_{2}$ samples (with $1 \mathrm{wt} . \% \mathrm{Pt}$ as co-catalyst, Supplementary Fig. 2) was measured using the amount of hydrogen production from water splitting under different irradiations, and the time course of hydrogen evolution is shown in Fig. 4. Under ultraviolet light (Fig. 4a), T-1 exhibits the highest mass-specific activity $\left(24.7 \mathrm{mmol} \mathrm{h}{ }^{-1} \mathrm{~g}^{-1}\right)$, followed by T-3 $\left(15.4 \mathrm{mmol} \mathrm{h}^{-1} \mathrm{~g}^{-1}\right)$ and then T-2 $\left(9.2 \mathrm{mmol} \mathrm{h}^{-1} \mathrm{~g}^{-1}\right)$. It is surprising to note that $\mathrm{T}-1$ with more defects exhibits distinctly higher photocatalytic activity than T-2 with less defects, considering that defects are generally regarded as the charge-carrier trapping and recombination centres. Under visible light $(400 \mathrm{~nm}<\lambda<780 \mathrm{~nm}$, Fig. $4 \mathrm{~b})$, T-1 exhibits the highest massspecific activity $\left(932 \mu \mathrm{mol} \mathrm{h}^{-1} \mathrm{~g}^{-1}\right)$, followed by $\mathrm{T}-2$ $\left(372 \mu \mathrm{molh}^{-1} \mathrm{~g}^{-1}\right)$ and then $\mathrm{T}-3\left(117 \mu \mathrm{molh}^{-1} \mathrm{~g}^{-1}\right)$, consistent with their ability to absorb the visible light. Indeed, the hydrogen evolution rate achieved on T-1 is much greater, that is, more than five times higher, than other $\mathrm{TiO}_{2}$-based photocatalysts ever reported. A direct comparison between $\mathrm{TiO}_{2}$-based materials for visible-light-driven hydrogen production is summarized in Supplementary Table 3. Under the irradiation of full-spectrum simulator, that is, sunlight air mass T-1 also exhibits a remarkable photocatalytic activity with a hydrogen evolution rate of $1,954 \mu \mathrm{mol} \mathrm{h}^{-1} \mathrm{~g}^{-1}, 4.2$ and 5.6 times higher than that of T-2 and T-3, respectively (Fig. 4c). This value is also more than three times higher than reference $\mathrm{TiO}_{2} \mathrm{P} 25$. For a more objective comparison between the photocatalytic efficiencies for hydrogen production under visible light, the apparent quantum yield (QY) was calculated. The highest QY of $3.52 \%$ is obtained on T-1 sample under monochromatic light at $\lambda=405$ $\mathrm{nm}$, followed by $1.40 \%$ of T-2 and $0.36 \%$ of T-3, consistent with the trend in photocatalytic activity under visible light (Fig. $4 \mathrm{~b}$ ). Moreover, high QY of $1.74 \%$ can be obtained on T-1 sample under monochromatic light at $\lambda=420 \mathrm{~nm}$. To our knowledge, these are the highest QYs ever reported for stable oxide semiconductors under comparable conditions ${ }^{18,53}$, although they are still lower than other systems, for example, CdSe nanocrystals capped with dihydrolipoic acid ${ }^{54}$ and Ni-decorated CdS nanorods ${ }^{55}$. 

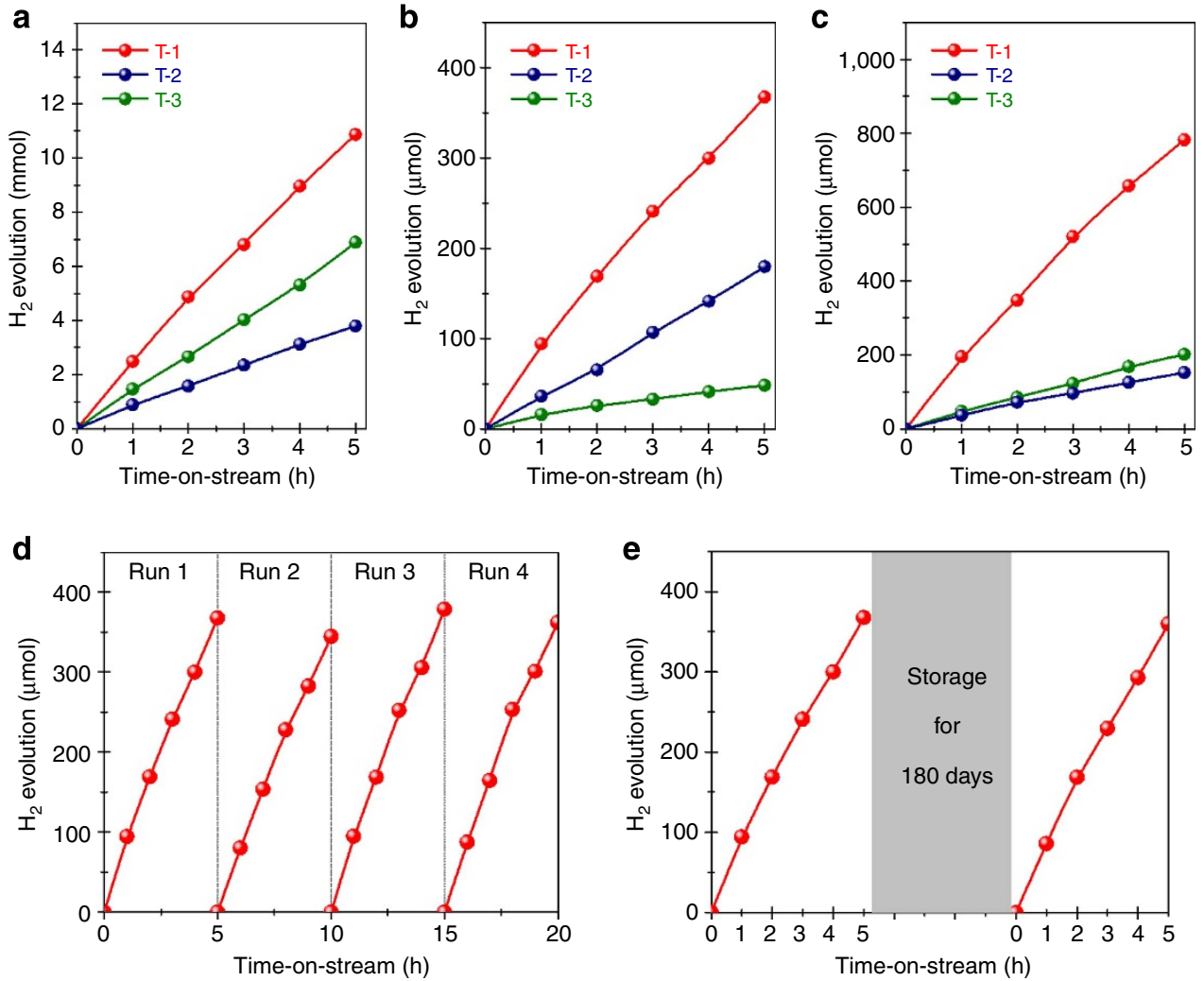

Figure 4 | Photocatalytic $\mathbf{H}_{\mathbf{2}}$ production over platinized rutile $\mathbf{T i O}_{\mathbf{2}}$. (a) Under ultraviolet light, $320 \mathrm{~nm}<\lambda<400 \mathrm{~nm}$. (b) Under visible light, $400 \mathrm{~nm}<\lambda<780 \mathrm{~nm}$. (c) Under sunlight air mass 1.5 irradiation. (d) Cycling tests of T-1 under visible light. (e) Photcatalytic activity of T-1 after long-term storage, under visible light. Pt (1 wt\%) is in situ photo-deposited on the surface of samples as the co-catalyst and $10 \mathrm{ml}$ methanol is used as sacrificial reagent for water splitting.

In addition to its remarkable photocatalytic activity, T-1 exhibits very good stability as a photocatalyst. As shown in Fig. 4d, no noticeable decrease in the activity for photocatalytic hydrogen production can be observed in the cycling tests. Moreover, no obvious activity loss can be observed for T-1 even after conventional storage (sealed in glass sample bottle with finger tight) for as long as 180 days (Fig. 4e). Therefore, the remarkable activity and stability make $\mathrm{T}-1$ a promising semiconductor photocatalyst for hydrogen production from water splitting under visible light or solar light.

Functionalities of defects in rutile $\mathrm{TiO}_{2}$ nanoparticles. For sub$10 \mathrm{~nm}$ rutile nanoparticles $\mathrm{T}-1$, the presence of abundant defects is clearly revealed by Raman, FTIR, XPS, photoluminescence and positron annihilation lifetime spectroscopy. The absence of reduced $\mathrm{Ti}$ states, for example, $\mathrm{Ti}^{3+}$, is confirmed by Ti $2 p$ XPS (Fig. 1c) and Ti $L_{2,3}$-edge EELS (Fig. 2d). Therefore, the existence of oxygen vacancies or $\mathrm{Ti}$ interstitials, which are considered as major defects in $\mathrm{TiO}_{2}$ contributing to band-gap states, could be expressly excluded. This is also supported by the fact that our rutile $\mathrm{TiO}_{2}$ sample is exposed to ambient conditions with water and oxygen that would easily heal the oxygen vacancies and reduced Ti sites. Meanwhile, the presence of bridging hydroxyls is confirmed by O 1-s XPS (Fig. 1d) and FTIR analysis (Supplementary Fig. 1). As the bridging hydroxyls are known to come from the dissociative adsorption of water on oxygen vacancies ${ }^{56,57}$, the detectable hydroxyls should indicate the everexisting oxygen vacancies, together with $\mathrm{Ti}^{3+}$. That is, oxygen vacancies could be created during the synthesis of sub-10 nm rutile $\mathrm{TiO}_{2}$ nanoparticles via fast hydrolysis, but they immediately react with water to form hydroxyls $\left(\mathrm{O}_{b}-\mathrm{vac}+\mathrm{O}_{b}+\mathrm{H}_{2} \mathrm{O} \rightarrow\right.$ $2 \mathrm{OH}_{b}$, where $\mathrm{O}_{b}-$ vac means bridging oxygen vacancy, $\mathrm{O}_{b}$ means bridging oxygen and $\mathrm{OH}_{b}$ means bridging hydroxyl). In this context, hydroxyls should be the major defects in sub-10 nm rutile $\mathrm{TiO}_{2}$ nanoparticles, also under ambient conditions, and the concentration of hydroxyls represents the defect degree in samples. The results from positron annihilation (Fig. 1f) and photoluminescence spectroscopy (Supplementary Fig. 3) further indicate the presence of similar types of surface defects, that is, hydroxyls, of rutile $\mathrm{TiO}_{2}$ prepared via both hydrolysis and hydrothermal routes. Thus, the only difference in rutile $\mathrm{TiO}_{2}$ samples under study lies in their defect degrees, which directly influence the amount of upward shift in the valence band top (Supplementary Table 1), and are responsible for the band-gap narrowing. The upward shift in the valence band top due to the existence of abundant defects in T-1 should be ascribed to the band bending ${ }^{30}$, most probably associated with charge imbalance induced by the defect energy levels ${ }^{58}$. Calcination at elevated temperature will eliminate some of the defects in T-1 and, therefore, reduce the extent of upward shift in the valence band top of T-2 (Fig. 3d).

On the other hand, the defects in $\mathrm{TiO}_{2}$ may greatly influence the efficiency of charge-carrier separation and the corresponding photocatalytic activity. The bulk defects will introduce chargecarrier trapping and recombination sites, and show degradation effects on the photocatalytic activity. In contrast, surface and subsurface defects may contribute to charge-carrier separation, as the charges at surface or shallow traps become available for photocatalytic reaction. For most $\mathrm{TiO}_{2}$-based semiconductor materials reported, for example, doped $\mathrm{TiO}_{2}$, high defect degrees play a negative role on the photocatalytic activity, because the 
number of bulk defects is generally much higher than that of surface and sub-surface defects.

As discussed above, more surface and sub-surface defects are desired for band-gap narrowing, while less bulk defects are wanted to promote the charge carrier separation. In general, the degrading effects from bulk defects will negate the increased visible light absorption from band-gap narrowing induced by surface defects and low visible-light-driven photocatalytic activity will be observed. In this study, we present a new concept to solve the problem by reducing the size of $\mathrm{TiO}_{2}$ nanoparticles. With decreasing particle size, the ratio of surface to bulk increases, indicating the increased ratio of surface/sub-surface to bulk defects (Fig. 1f). Band-gap broadening due to quantum size effect, expressed as equation (2), could be neglected with $\mathrm{TiO}_{2}$ particle size of $>3 \mathrm{~nm}^{59,60}$.

$$
\Delta \mathrm{E}=\frac{h^{2} \pi^{2}}{2 R^{2}}\left|\frac{1}{m_{\mathrm{e}}}+\frac{1}{m_{\mathrm{h}}}\right|-\frac{1.786 e^{2}}{\varepsilon R}-0.248 E_{\mathrm{Ry}}^{*}
$$

For T-1 with an average diameter of $c a .8 \mathrm{~nm}$ employed in this study (Fig. 2a), the positive effects from surface/sub-surface defects is strong enough to overcome the negative effects from bulk defects due to the relative high percentage of surface/subsurface. This is well confirmed by its remarkable photocatalytic activity in hydrogen production under ultraviolet light (Fig. 4a).
Taking the functionalities of defects on both band-gap narrowing and charge-carrier separation into consideration, we successfully develop the sub-10 nm rutile $\mathrm{TiO}_{2}$ nanoparticles for efficient visible-light-driven photocatalytic hydrogen production. The visible light response of sub- $10 \mathrm{~nm}$ rutile $\mathrm{TiO}_{2}$ nanoparticles is first created via the introduction of abundant defects and then promoted by the specific defect distribution in nanoparticles and the use of co-catalyst $\mathrm{Pt}$ as well. If rutile $\mathrm{TiO}_{2}$ with smaller particles size can be synthesized, higher visible-light-driven photocatalytic activity could be expected.

Role of $\mathrm{Ti}^{3+}$ in photocatalytic hydrogen production. The role of $\mathrm{Ti}^{3+}$ in the visible-light photocatalytic activity of $\mathrm{TiO}_{2}$-based materials has been intensively debated in the literature ${ }^{17,20-24,61}$. The major issue is that the appearance of $\mathrm{Ti}^{3+}$ is always accompanied by defects and, therefore, will inevitably compound the problem. For our as-prepared rutile $\mathrm{TiO}_{2}$ samples, the existence of lower oxidation states of $\mathrm{Ti}$, that is, $\mathrm{Ti}^{3+}$, can be excluded based on XPS analysis. However, the presence of $\mathrm{Ti}^{3+}$ can be clearly observed for all rutile samples on ultraviolet irradiation, as confirmed by the distinct electron spin resonance (ESR) signal at $g_{1}=1.972$ and $g_{2}=1.949$ (Fig. 5a) ${ }^{20,62}$. We then measured the quasi in situ ultraviolet-visible spectra of T-1 and T-3 for further information. As shown in Fig. 5b, the presence of a
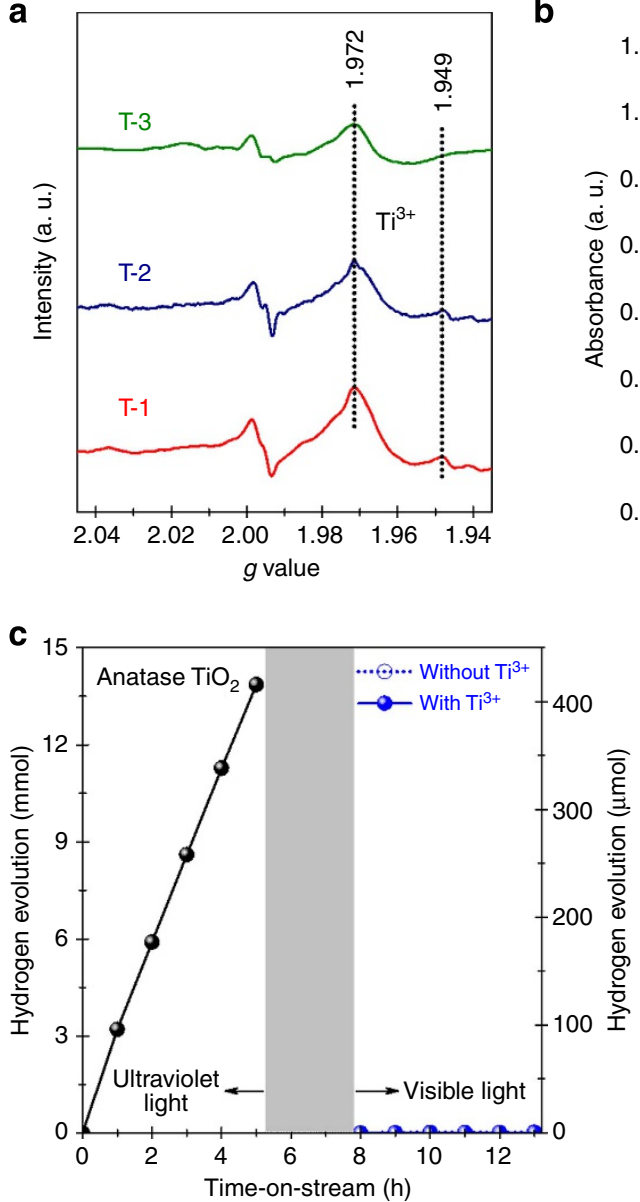

b
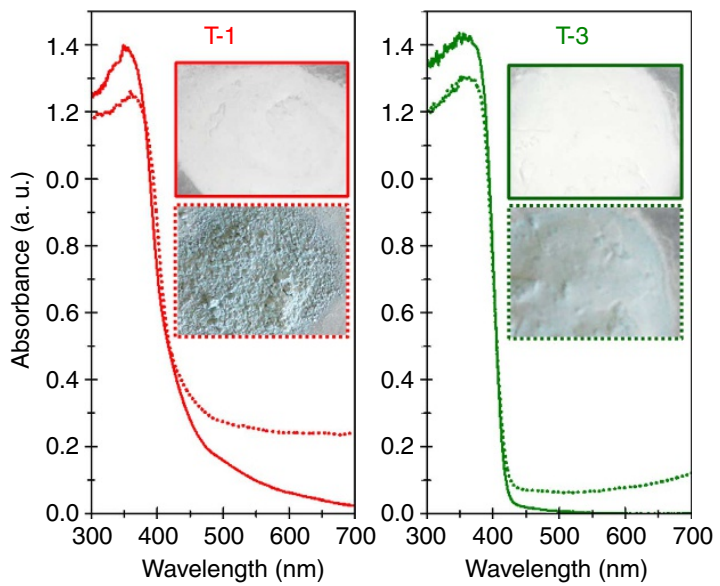

d

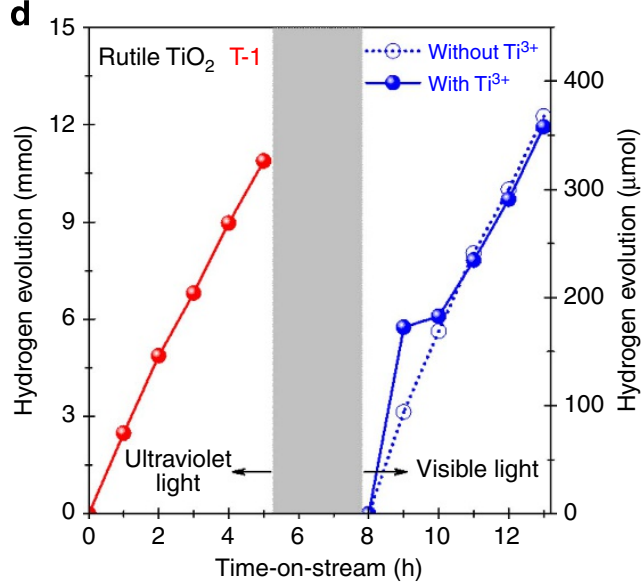

Figure 5 | Existence and role of $\mathbf{T i}^{\mathbf{3}+}$ in photocatalytic hydrogen production. (a) ESR spectra of rutile $\mathrm{TiO}_{2}$ samples under ultraviolet irradiation. (b) Quasi in situ ultraviolet-visible spectra of platinized rutile $\mathrm{TiO}_{2}$ during photocatalytic hydrogen production from water splitting under ultraviolet irradiation; solid line: ultraviolet-visible spectrum before irradiation; dashed line: ultraviolet-visible spectrum after ultraviolet irradiation for 5 min; inset: photographs of samples before and after $5 \mathrm{~min}$ reaction). (c,d) Step responses of photocatalytic activity of platinized anatase and rutile $\mathrm{TiO}_{2}$ to the change of irradiation light from ultraviolet light $(400 \mathrm{~nm}>\lambda>320 \mathrm{~nm})$ to visible light $(780 \mathrm{~nm}>\lambda>400 \mathrm{~nm})$. 
$\mathrm{Ti}^{3+}$ can be verified by the additional absorption in the visible light region for both $\mathrm{T}-1$ and $\mathrm{T}-3$ after 5 min time-on-stream ultraviolet irradiation to create a sufficiently detectable amount of $\mathrm{Ti}^{3}+$ (ref. 63). Judging from the intensity of absorption intensity, we propose that more $\mathrm{Ti}^{3+}$ species are presented in sub-10 nm rutile $\mathrm{TiO}_{2}$ nanoparticles $\mathrm{T}-1$ than reference $\mathrm{T}-3$ on ultraviolet irradiation, which is clearly confirmed by the different intensities of $\mathrm{Ti}^{3+}$ signals in ESR spectra (Fig. 5a). That is, the presence of defects in rutile $\mathrm{TiO}_{2}$ should facilitate $\mathrm{Ti}^{3+}$ formation during photocatalytic reaction under ultraviolet irradiation.

To obtain a direct evidence on the role of $\mathrm{Ti}^{3+}$ in the photocatalytic hydrogen production, step-response photocatalytic experiments are performed. Under ultraviolet light, anatase $\mathrm{TiO}_{2}$ nanoparticles (Supplementary Fig. 4) are highly active in photocatalytic water splitting with a hydrogen evolution rate of $31.2 \mathrm{mmolh}^{-1} \mathrm{~g}^{-1}$ (Fig. 5c), slightly higher than the $24.7 \mathrm{mmol} \mathrm{h}^{-1} \mathrm{~g}^{-1}$ from sub-10 nm rutile $\mathrm{TiO}_{2}$ nanoparticles (Fig. 5d). During photocatalytic reaction under ultraviolet irradiation, the presence of $\mathrm{Ti}^{3+}$ is quite obvious with blue colouration (inset of Fig. 5b and Supplementary Fig. 5) ${ }^{63,64}$. After a 5 -h photocatalytic reaction under ultraviolet radiation, the reaction system was either evacuated to preserve $\mathrm{Ti}^{3+}$ or exposed to oxygen and then evacuated to eliminate $\mathrm{Ti}^{3+}$. In both cases, the anatase $\mathrm{TiO}_{2}$ does not exhibit any detectable activity in subsequent photocatalytic hydrogen production tests under visible light (Fig. 5c). Therefore, it is rational to propose that the existence of $\mathrm{Ti}^{3+}$ itself will not initiate the visible light photocatalytic activity for hydrogen production. This is consistent with the fact that the band-gap state created by $\mathrm{Ti}^{3}+3 d^{1}$ state is about $1.0 \mathrm{eV}$ below the conduction band of $\mathrm{TiO}_{2}$ (ref. 32), which is obviously not suitable for $\mathrm{H}_{2} \mathrm{O}$ reduction to hydrogen (Fig. 2e). Sub-10 $\mathrm{nm}$ rutile $\mathrm{TiO}_{2}$, that is, $\mathrm{T}-1$, is treated in the same manner and it exhibits remarkable photocatalytic activity under visible light after both treatments (Fig. 5d), which is ascribed to the surface defects, that is, hydroxyls, enabling the upward shift of valence band top of rutile $\mathrm{TiO}_{2}$. We also observe that $\mathrm{T}-1$ with a significant $\mathrm{Ti}^{3+}$ concentration exhibits much higher initial visible-light photocatalytic activity than that without $\mathrm{Ti}^{3+}$ (time-on-stream of $1 \mathrm{~h}$, hydrogen evolution rate from 967 to $1,763 \mu \mathrm{mol} \mathrm{h}^{-1} \mathrm{~g}^{-1}$ ). The photocatalytic activity becomes almost the same after a time-on-stream of $2 \mathrm{~h}$. The presence of $\mathrm{Ti}^{3+}$ can enhance the initial visible-light photocatalytic activity most probably due to the promoted methanol adsorption and/or the enhanced light absorbance and scattering ${ }^{65}$, and the promotional effects gradually fade away with the elimination of $\mathrm{Ti}^{3+}$. Consequently, we conclude that the presence of $\mathrm{Ti}^{3+}$ cannot initiate visible-light photocatalytic activity in hydrogen production, but $\mathrm{Ti}^{3+}$ is able to enhance the visible-light photocatalytic activity due to the promoted methanol adsorption.

\section{Discussion}

In summary, we have successfully developed a simple and scalable hydrolysis route to sub- $10 \mathrm{~nm}$ rutile $\mathrm{TiO}_{2}$ nanoparticles with diameters below $10 \mathrm{~nm}$. We achieve a state-of-the-art hydrogen evolution rate of $932 \mu \mathrm{molh}^{-1} \mathrm{~g}^{-1}$ under visible light $(>400 \mathrm{~nm})$ and $1,954 \mu \mathrm{mol} \mathrm{h}^{-1} \mathrm{~g}^{-1}$ under simulated solar light with platinized sub- $10 \mathrm{~nm}$ rutile $\mathrm{TiO}_{2}$ nanoparticles ( $1 \mathrm{wt} . \% \mathrm{Pt}$ ) among $\mathrm{TiO}_{2}$-based semiconductors. Spectroscopic characterization results clearly confirm the existence of abundant surface defects, that is, hydroxyls, in the as-prepared rutile $\mathrm{TiO}_{2}$ nanoparticles, which is responsible for the band-gap narrowing. The sub-10 nm particle size increases the percentage of surface/ sub-surface defects compared with bulk defects, which results in enhanced charge-carrier separation. Moreover, we have revealed that the existence of defects in rutile $\mathrm{TiO}_{2}$ is in favour of $\mathrm{Ti}^{3+}$ formation during the photocatalytic reaction under ultraviolet irradiation. The presence of $\mathrm{Ti}^{3+}$ cannot initiate the visible-light photocatalytic activity, but it is able to enhance the visible-lightdriven water splitting most likely due to the promoted reagent adsorption. The results presented here provide new insights into the functionalities of defects in visible-light photocatalytic activity. The concept of ultra-small nanoparticles should be useful for the future design of robust semiconductor photocatalysts with tunable band gap.

\section{Methods}

Preparation of rutile $\mathrm{TiO}_{2}$. All of the chemical reagents of analytical grade were purchased from Alfa Aesar Chemical Co. and used as received without further purification. In a typical hydrolysis synthesis of rutile $\mathrm{TiO}_{2}, 10 \mathrm{ml}$ titanium tetrachloride $\left(\mathrm{TiCl}_{4}\right)$ was dropwise added into $30 \mathrm{ml}$ ice water under stirring to prepare a transparent $\mathrm{TiCl}_{4}$ aqueous solution. After further stirring for $30 \mathrm{~min}$, the $\mathrm{TiCl}_{4}$ aqueous solution was rapidly heated to $373 \mathrm{~K}$ (within $5 \mathrm{~min}$ ) to remove the water and hydrogen chloride (Note: Rapid heating is crucial to obtain rutile $\mathrm{TiO}_{2}$ nanoparticles). The obtained white solid was thoroughly washed with deionized water, followed by drying in air at $353 \mathrm{~K}$ for $24 \mathrm{~h}$ and calcination in a muffle furnace at temperatures of 473 or $673 \mathrm{~K}$ for $2 \mathrm{~h}$. The final products are denoted as T-1 and T-2, respectively. For reference, rutile $\mathrm{TiO}_{2}$ was also prepared via a hydrothermal route. Typically, $10 \mathrm{ml}$ of $1 \mathrm{M} \mathrm{TiCl}_{4}$ aqueous solution was added to $50 \mathrm{ml}$ water and the resulting solution was directly transferred into a $75-\mathrm{ml}$ Teflonlined autoclave for static crystallization at $453 \mathrm{~K}$ for $24 \mathrm{~h}$. The resulting precipitates after crystallization were separated from the liquid phase by centrifugation, thoroughly washed with water, dried at $353 \mathrm{~K}$ for $24 \mathrm{~h}$, subjected to calcination at $473 \mathrm{~K}$ for $2 \mathrm{~h}$ and denoted as $\mathrm{T}-3$. For the synthesis of anatase $\mathrm{TiO}_{2}, \mathrm{TiCl}_{4}$ was dropwise added into ice water under stirring to prepare a $\mathrm{TiCl}_{4}$ aqueous solution with concentration of $1 \mathrm{moll}^{-1}$. Next, $30 \mathrm{ml}$ of $\mathrm{TiCl}_{4}$ aqueous solution was mixed with $30 \mathrm{ml}$ of $\mathrm{KOH}$ solution $\left(1 \mathrm{moll}^{-1}\right)$ and the resulting solution was transferred into a 75-ml Teflon-lined autoclave for static crystallization at $373 \mathrm{~K}$ for $24 \mathrm{~h}$. The resulting precipitates after crystallization were separated from the liquid phase by centrifugation, thoroughly washed with water, dried at $353 \mathrm{~K}$ for $24 \mathrm{~h}$, subjected to calcination at $473 \mathrm{~K}$ for $2 \mathrm{~h}$ and denoted as T-4.

Characterization techniques. The specific surface areas of $\mathrm{TiO}_{2}$ samples were determined through $\mathrm{N}_{2}$ adsorption/desorption isotherms at $77 \mathrm{~K}$ collected on a Quantachrome iQ-MP gas adsorption analyser. The X-ray diffraction patterns of $\mathrm{TiO}_{2}$ samples were recorded on a Bruker D8 ADVANCE powder diffractometer using $\mathrm{Cu}-\mathrm{K} \alpha$ radiation $(\lambda=0.1542 \mathrm{~nm})$ at a scanning rate of $4^{\circ}$ per min in the region of $2 \theta=20-80^{\circ}$. Raman analysis was carried out on a Renishaw InVia Raman spectrometer and the spectra were obtained with the green line of an Ar-ion laser $(514.53 \mathrm{~nm})$ in micro-Raman configuration. Diffuse reflectance ultravioletvisible spectra of $\mathrm{TiO}_{2}$ samples (ca. $20 \mathrm{mg}$ diluted in $c a .80 \mathrm{mg} \mathrm{BaSO}$ ) were recorded in the air against $\mathrm{BaSO}_{4}$ in the region of $200-700 \mathrm{~nm}$ on a Varian Cary 300 ultraviolet-visible spectrophotometer. For the so-called quasi in situ ultraviolet-visible spectroscopy, $50 \mathrm{mg}$ platinized $\mathrm{TiO}_{2}(1 \mathrm{wt} . \% \mathrm{Pt})$ was mixed with $10 \mathrm{ml}$ methanol aqueous solution $(10 \%)$ in a home-made quartz reaction chamber, evacuated and then sealed for analysis. The diffuse reflectance ultraviolet-visible spectra against $\mathrm{BaSO}_{4}$ were recorded before and after ultraviolet light irradiation for $10 \mathrm{~min}$ on the Varian Cary 300 ultraviolet-visible spectrophotometer. The concentration of surface hydroxyls in rutile $\mathrm{TiO}_{2}$ was analysed by thermogravimetry on a Setram Setsys $16 / 18$ thermogravmetric analyser. In a typical measurement, $0.1 \mathrm{~g}$ of rutile $\mathrm{TiO}_{2}$ sample was heated in an $\mathrm{Al}_{2} \mathrm{O}_{3}$ crucible from 300 to $973 \mathrm{~K}$ with a constant heating rate of $10 \mathrm{~K} \mathrm{~min}^{-1}$ and under flowing Ar of $20 \mathrm{ml} \mathrm{min}^{-1}$. TEM images were taken on a FEI Tecnai G2 F20 electron microscope at an acceleration voltage of $200 \mathrm{kV}$ and a Tecnai G2 F30 electron microscope at an acceleration voltage of $300 \mathrm{kV}$. A few drops of alcohol suspension containing the sample were placed on a carbon-coated copper grid, followed by evaporation at ambient temperature. EELS spectra of samples were collected using a GIF Tridiem 863 analyser. The lattice structural analysis was done using the Digital Micrograph software. XPS spectra of samples were recorded on a Kratos Axis Ultra delay line detector spectrometer with a monochromated Al-K $\alpha$ X-ray source ( $h v=1486.6$ $\mathrm{eV})$, hybrid (magnetic/electrostatic) optics and a multi-channel plate and delay line detector. All spectra were recorded using an aperture slot of $300 \times 700 \mu \mathrm{m}$. Survey spectra were recorded with a pass energy of $160 \mathrm{eV}$ and high-resolution spectra with a pass energy of $40 \mathrm{eV}$. Accurate binding energies $( \pm 0.1 \mathrm{eV})$ were determined with respect to the position of the adventitious C $1 \mathrm{~s}$ peak at $284.8 \mathrm{eV}$. VB XPS of samples were measured on PHI Quantera XPS Scanning Microprobe spectrometer using $\mathrm{Al}-\mathrm{K} \alpha \mathrm{X}$-ray source $(\mathrm{h} \nu=1486.6 \mathrm{eV})$. The energy scales are aligned by using the Fermi level of the XPS instrument $(4.10 \mathrm{eV}$ versus absolute vacuum value). Mott-Schottky plots were obtained using a three-electrode cell electrochemical workstation (IVIUM CompactStat). The saturated $\mathrm{Ag} / \mathrm{AgCl}$ and platinum foil $\left(2 \times 2 \mathrm{~cm}^{2}\right)$ were used as the reference electrode and the counter electrode,

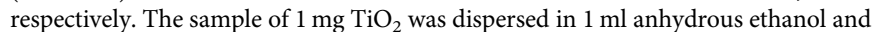
then evenly grinded to a slurry. The slurry was spread onto indium tin oxide glass and the exposed area was kept at $0.25 \mathrm{~cm}^{2}$. The prepared indium tin oxide/samples 
were dried overnight under ambient conditions and then used as the working electrode. The measurements were carried out at a fixed frequency of $1 \mathrm{kHz}$ in $0.5 \mathrm{M} \mathrm{Na}_{2} \mathrm{SO}_{4}$ solution in the dark. Positron annihilation experiments were performed on a fast-slow coincidence ORTEC system with a time resolution of $187 \mathrm{ps}$ full width at half maximum. The sample powder was pressed into a disk (diameter: $10.0 \mathrm{~mm}$, thickness: $1.0 \mathrm{~mm}$ ). A $5 \times 10^{5}-\mathrm{Bq}$ source of ${ }^{22} \mathrm{Na}$ was sandwiched between two identical sample disks. Measured spectra were analysed by the computer programme LT9.0, with source correction to evaluate the lifetime component $\tau_{\mathrm{i}}$ and corresponding intensity $I_{\mathrm{i}}$, using equation (3):

$$
N(t)=\sum_{i=1}^{k+1} \frac{I_{\mathrm{i}}}{\tau_{\mathrm{i}}} \exp \left(-\frac{t}{\tau_{\mathrm{i}}}\right)
$$

ESR was carried out on a JEOL JES-FA200 cw-EPR spectrometer (X-band) with a microwave power of $1.0 \mathrm{~mW}$ and a modulation frequency of $100 \mathrm{kHz}$. MnMarker was used as an internal standard for the measurement of the magnetic field. In a typical experiment, sample of $150 \mathrm{mg}$ was placed in a quartz ESR tube and evacuated at room temperature for $2 \mathrm{~h}$. After cooling down to $110 \mathrm{~K}$, the tube was analysed under ultraviolet irradiation.

Photocatalytic hydrogen production from water splitting. Photocatalytic water splitting with methanol as sacrificial agent was performed in a top-irradiation-type Pyrex reaction cell connected to a closed gas circulation and evacuation system under the irradiation of Xe lamp (PLS-SXE, wavelength: $300-2,500 \mathrm{~nm}$ ) with different optical reflector or filter (UVREF: $320-400 \mathrm{~nm}, c a .83 \mathrm{~mW} \mathrm{~cm}^{-2}$; UVCUT400: $\left.400-780 \mathrm{~nm}, c a .80 \mathrm{~mW} \mathrm{~cm}^{-2}\right)$ or Air Mass $1.5\left(c a .100 \mathrm{~mW} \mathrm{~cm}^{-2}\right)$. In a typical experiment, catalyst sample of $100 \mathrm{mg}$ was suspended in $c a .100 \mathrm{ml} 10 \%$ methanol aqueous solution $\left(10 \mathrm{ml}\right.$ methanol and $\left.90 \mathrm{ml} \mathrm{H}_{2} \mathrm{O}\right)$ in the reaction cell. After being evacuated for $30 \mathrm{~min}$, the reactor cell was irradiated by the Xe lamp at $200 \mathrm{~W}$, at a constant temperature of $313 \mathrm{~K}$ under stirring. The gaseous products were analysed by an on-line gas chromatograph (Varian CP-3800) with a thermal conductivity detector. For all $\mathrm{TiO}_{2}$ samples, 1 wt.\% $\mathrm{Pt}$ was in situ photo-deposited from $1 \mathrm{M} \mathrm{H}_{2} \mathrm{PtCl}_{6}$ aqueous solution as co-catalyst, resulting a $\mathrm{pH}$ value of 6.5 in the reaction system. The apparent QYwas measured using the same experimental setup for the photocatalytic hydrogen generation, but with additional band-pass filters to obtain monochromatic light at $\lambda=405 \mathrm{~nm}$ or $\lambda=420 \mathrm{~nm}$ (together with UVCUT400). The power density was measured to be $c a .2 .0(\lambda=405 \mathrm{~nm})$ or $1.5 \mathrm{~mW} \mathrm{~cm}^{-2}(\lambda=420 \mathrm{~nm})$ using a calibrated photodiode and the QY was calculated by equation (4):

$$
\mathrm{QY}=\frac{\text { Number of reacted electrons }}{\text { Number of incident photons }} * 100 \%=\frac{\text { Number of evolved } \mathrm{H}_{2} \text { molecules } * 2}{\text { Number of incident photons }} * 100 \%
$$

DFT calculations. DFT calculations were performed with the plane-wave-based Vienna $a b$ initio simulation package VASP ${ }^{66}$ at hybrid level in form of HSE06 (ref. 67). The $(2 \times 2)$ rutile $\mathrm{TiO}_{2}(100)$ was represented by periodic slab models of four tri- $\mathrm{TiO}_{2}$ layer thick. The valence wave functions were expanded in a planewave basis with a cutoff energy of $400 \mathrm{eV}$, while the interaction between the atomic cores and the electrons was described by the projector augmented wave method ${ }^{68}$. A Monkhorst-Pack mesh ${ }^{69}$ of $2 \times 1 \times 1$ was used to sample the Brillouin zone during the geometry optimization (to $5 \times 10^{-4} \mathrm{eV} \mathrm{pm}^{-1}$ ), while the mesh was increased to $4 \times 2 \times 1$ in density of state analysis. The bands of different defect slabs were aligned to the same vacuum level.

\section{References}

1. Fujishima, A. \& Honda, K. Electrochemical photolysis of water at a semiconductor electrode. Nature 238, 37-38 (2008).

2. Hoffmann, M. et al. Environmental applications of semiconductor photocatalysis. Chem. Rev. 95, 69-96 (1995).

3. Thompson, L. \& Yates, J. Surface science studies of the photoactivation of $\mathrm{TiO}_{2}$-new photochemical processes. Chem. Rev. 106, 4428-4453 (2006).

4. Ravelli, D. et al. Photocatalysis. A multi-faceted concept for green chemistry. Chem. Soc. Rev. 39, 1999-2011 (2009).

5. Hernandez-Alonso, M. et al. Development of alternative photocatalysts to $\mathrm{TiO}_{2}$ : challenges and opportunities. Energy Environ. Sci. 2, 1231-1257 (2009).

6. Chen, X. \& Mao, S. S. Titanium dioxide nanomaterials: synthesis, properties, modifications and application. Chem. Rev. 107, 2891-2959 (2007).

7. Asahi, R. et al. Visible-light photocatalysis in nitrogen-doped titanium oxides. Science 293, 269-271 (2001)

8. Khan, S. U., Al-Shahry, M. \& Ingler, W. B. Efficient photochemical water splitting by a chemically modified $\mathrm{n}-\mathrm{TiO}_{2}$. Science 297, 2243-2245 (2002).

9. Sakthivel, S. \& Kisch, H. Daylight photocatalysis by carbon-modified titanium dioxide. Angew. Chem. Int. Ed. 42, 4908-4911 (2003).

10. Park, J. H., Kim, S. \& Bard, A. J. Novel carbon-doped $\mathrm{TiO}_{2}$ nanotube arrays with high aspect ratios for efficient solar water splitting. Nano Lett. 6, 24-28 (2006).

11. Hoang, S. et al. Visible light driven photoelectrochemical water oxidation on nitrogen-modified $\mathrm{TiO}_{2}$ nanowires. Nano Lett. 12, 26-32 (2002).
12. Zhao, W. et al. Efficient degradation of toxic organic pollutants with $\mathrm{Ni}_{2} \mathrm{O}_{3}$ / $\mathrm{TiO}_{2-\mathrm{x}} \mathrm{B}_{\mathrm{x}}$ under visible irradiation. J. Am. Chem. Soc. 126, 4782-4783 (2004).

13. Umebayashi, T. et al. Band gap narrowing of titanium dioxide by sulfur doping. Appl. Phys. Lett. 81, 454-456 (2002).

14. Borgarello, E. et al. Visible light induced water cleavage in colloidal solutions of chromium-doped titanium dioxide particles. J. Am. Chem. Soc. 104, 2996-3002 (1982).

15. Liu, M. et al. Energy-level matching of Fe(III) ions grafted at surface and doped in bulk for efficient visible-light photocatalysts. J. Am. Chem. Soc. 135, 10064-10072 (2013).

16. Chen, X. \& Burda, C. The electronic origin of the visible-light absorption properties of C-, N- and S-doped $\mathrm{TiO}_{2}$ nanomaterials. J. Am. Chem. Soc. 130, 5018-5019 (2008).

17. Hoang, S. et al. Enhancing visible light photo-oxidation of water with $\mathrm{TiO}_{2}$ nanowire arrays via cotreatment with $\mathrm{H}_{2}$ and $\mathrm{NH}_{3}$ : synergistic effects between $\mathrm{Ti}^{3+}$ and N. J. Am. Chem. Soc. 134, 3659-3662 (2012).

18. Chen, X. et al. Semiconductor-based photocatalytic hydrogen generation. Chem. Rev. 110, 6503-6570 (2010).

19. Justicia, I. et al. Designed self-doped titanium oxide thin films for efficient visible-light photocatalysis. Adv. Mater. 14, 1399-1402 (2002).

20. Zuo, F. et al. Self-doped $\mathrm{Ti}^{3+}$ enhanced photocatalyst for hydrogen production under visible light. J. Am. Chem. Soc. 132, 11856-11857 (2010).

21. Zuo, F. et al. Active facets on titanium (III)-doped $\mathrm{TiO}_{2}$ : an effective strategy to improve the visible-light photocatalytic activity. Angew. Chem. Int. Ed. 124, 6223-6226 (2012).

22. Chen, X. et al. Increasing solar absorption for photocatalysis with black hydrogenated titanium dioxide nanocrystals. Science 331, 746-750 (2011).

23. Naldoni, A. et al. Effect of nature and location of defects on bandgap narrowing in black $\mathrm{TiO}_{2}$ nanoparticles. J. Am. Chem. Soc. 134, 7600-7603 (2012).

24. Chen, X. et al. Properties of disorder-engineered black titanium dioxide nanoparticles through hydrogenation. Sci. Rep. 3, 1510 (2013).

25. Zheng, Z. et al. Hydrogenated titania: synergy of surface modification and morphology improvement for enhanced photocatalytic activity. Chem. Commun. 48, 5733-5735 (2012).

26. $\mathrm{Hu}, \mathrm{Y}$. Highly efficient photocatalyst-hydrogenated black $\mathrm{TiO}_{2}$ for solar splitting of water to hydrogen. Angew. Chem. Int. Ed. 51, 2-5 (2012).

27. Wang, Z. et al. Visible-light photocatalytic, solar thermal and photoelectrochemical properties of aluminium-reduced black titania. Energy Environ. Sci. 6, 3007-3014 (2013).

28. Yang, C. et al. Core-shell nanostructured "Black" rutile titania as excellent catalyst for hydrogen production enhanced by sulfur doping. J. Am. Chem. Soc. 135, 17831-17838 (2013).

29. Wang, G. et al. Hydrogen-treated $\mathrm{TiO}_{2}$ nanowire arrays for photoelectrochemical water splitting. Nano Lett. 11, 3026-3033 (2011).

30. Tao, J., Luttrell, T. \& Batzill, M. A Two-dimensional phase of $\mathrm{TiO}_{2}$ with a reduced bandgap. Nat. Chem. 3, 296-300 (2011).

31. Ariga, H. et al. Surface-mediated visible-light photo-oxidation on pure $\mathrm{TiO}_{2}$ (001). J. Am. Chem. Soc. 131, 14670-14672 (2009).

32. Di Valentin, C. \& Pacchioni, G. Electronic structure of defect states in hydroxylated and reduced rutile $\mathrm{TiO}_{2}$ (110) surfaces. Phys. Rev. Lett. 97, 166803 (2006).

33. Hosono, E. et al. Growth of submicrometer-scale rectangular parallelepiped rutile $\mathrm{TiO}_{2}$ films in aqueous $\mathrm{TiCl}_{3}$ solutions under hydrothermal conditions. J. Am. Chem. Soc. 126, 7790-7791 (2004).

34. Aruna, S. T., Tirosh, S. \& Zaban, A. Nanosize rutile titania particle synthesis via a hydrothermal method without mineralizers. J. Mater. Chem. 10, 2388-2391 (2000).

35. Yurdakal, S. et al. Nanostructured rutile $\mathrm{TiO}_{2}$ for selective photocatalytic oxidation of aromatic alcohols to aldehydes in water. J. Am. Chem. Soc. 130, 1568-1569 (2008).

36. Li, Y., Fan, Y. \& Chen, Y. A novel method for preparation of nanocrystalline rutile $\mathrm{TiO}_{2}$ powders by liquid hydrolysis of $\mathrm{TiCl}_{4}$. J. Mater. Chem. 12, 1387-1390 (2002).

37. Wang, W. et al. Synthesis of rutile $\left(\alpha-\mathrm{TiO}_{2}\right)$ nanocrystals with controlled size and shape by low-temperature hydrolysis: effects of solvent composition. J. Phys. Chem. B 108, 14789-14792 (2004).

38. Ohsaka, T., Izumi, F. \& Fujiki, Y. Raman spectrum of anatase, $\mathrm{TiO}_{2}$. J. Raman Spectr. 7, 321-324 (1978).

39. Gupta, S. K. et al. Titanium dioxide synthesized using titanium chloride: size effect study using Raman spectroscopy and photoluminescence. J. Raman Spectr. 41, 350-355 (2010).

40. Scanlon, D. O. et al. Band alignment of rutile and anatase $\mathrm{TiO}_{2}$. Nat. Mater. 12, 798-801 (2013).

41. Sham, T. K. \& Lazarus, M. S. X-ray photoelectron spectroscopy (XPS) studies of clean and hydrated $\mathrm{TiO}_{2}$ (rutile) surfaces. Chem. Phys. Lett. 68, 426-432 (1979)

42. Ketteler, G. et al. The nature of water nucleation sites on $\mathrm{TiO}_{2}(110)$ surfaces revealed by ambient pressure X-ray photoelectron spectroscopy. J. Phys. Chem. C 111, 8278-8282 (2007). 
43. Krause-Rehberg, R. \& Leipner, H. S. Positron Annihilation in Semiconductors (Springer, 1999).

44. Dutta, S. et al. Defect dynamics in annealed $\mathrm{ZnO}$ by positron annihilation spectroscopy. J. Appl. Phys. 98, 053513 (2005).

45. Jiang, X. et al. Characterization of oxygen vacancy associates within hydrogenated $\mathrm{TiO}_{2}$ : a positron annihilation study. J. Phys. Chem. C 116, 22619-22624 (2012).

46. Kong, M. et al. Tuning the relative concentration ratio of bulk defects to surface defects in $\mathrm{TiO}_{2}$ nanocrystals leads to high photocatalytic efficiency. J. Am. Chem. Soc. 133, 16414-16417 (2012).

47. Liu, X. et al. Oxygen vacancy clusters promoting reducibility and activity of ceria nanorods. J. Am. Chem. Soc. 131, 3140-3141 (2009).

48. Henrich, V. E. \& Cox, A. F. The Surface Science of Metal Oxides (Cambridge University Press, 1993).

49. Yaoshiya, M., Tanaka, I., Kaneko, K. \& Adachi, H. First principles calculation of chemical shifts in ELNES/NEXAFS of titanium oxides. J. Phys. Condens. Mater. 11, 3217-3228 (1999).

50. Wang, C. M. et al. Crystal and electronic structure of lithiated nanosized rutile $\mathrm{TiO}_{2}$ by electron diffraction and electron energy-loss spectroscopy. Appl. Phys. Lett. 94, 233116 (2009).

51. Diebold, U. The surface science of titanium dioxide. Surf. Sci. Rep. 48, 53-229 (2003).

52. $\mathrm{Su}, \mathrm{F}$. et al. Dendritic $\mathrm{Au} / \mathrm{TiO}_{2}$ nanorod arrays for visible-light driven photoelectrochemical water splitting. Nanoscale 5, 9001-9009 (2013).

53. Osterloh, F. E. Inorganic materials as catalysts for photochemical splitting of water. Chem. Mater. 20, 35-54 (2008).

54. Han, Z. et al. Robust photogeneration of $\mathrm{H}_{2}$ in water using semiconductor nanocrystals and a nickel catalyst. Science 238, 1321-1324 (2012).

55. Simon, T. et al. Redox shuttle mechanism enhances photocatalytic $\mathrm{H}_{2}$ generation on Ni-decorated CdS nanorods. Nat. Mater. 13, 1013-1018 (2014).

56. Bikondoa, O. et al. Direct visualization of defect-mediated dissociation of water on $\mathrm{TiO}_{2}$ (110). Nat. Mater. 5, 189-192 (2006).

57. Pang, C. L., Lindsay, R. \& Thornton, G. Chemical reactions on rutile $\mathrm{TiO}_{2}(110)$. Chem. Soc. Rev. 37, 2328-2353 (2008).

58. Holt, D. B. \& Yacobi, B. G. Extended Defects in Semiconductors (Cambridge University Press, 2007).

59. Brus, L. Quantum crystallites and nonlinear optics. Appl. Phys. A 53, 465-474 (1991).

60. Kormann, C., Bahnemann, D. W. \& Hoffmann, M. R. Preparation and characterization of quantum-size titanium dioxide. J. Phys. Chem. 92, 5196-5201 (1988)

61. Xing, M. et al. Self-doped $\mathrm{Ti}^{3+}$-enhanced $\mathrm{TiO}_{2}$ nanoparticles with a highperformance photocatalysis. J. Catal. 297, 236-243 (2013).

62. Fenoglio, I., Greco, G., Livraghi, S. \& Fubini, B. Non-UV-induced radical reactions at the surface of $\mathrm{TiO}_{2}$ nanoparticles that may trigger toxic responses. Chem. Eur. J. 15, 4614-4621 (2009).
63. Ma, J. et al. Photohole trapping induced platinum cluster nucleation on the surface of $\mathrm{TiO}_{2}$ nanoparticles. J. Phys. Chem. C 118, 1111-1117 (2014).

64. Yan, J. et al. Understanding the effect of surface/bulk defects on the photocatalytic activity of $\mathrm{TiO}_{2}$ : anatase versus rutile. Phys. Chem. Chem. Phys. 15, 10978-10988 (2013).

65. Pang, C. L., Lindsay, R. \& Thornton, G. Structure of clean and adsorbatecovered single-crystal rutile $\mathrm{TiO}_{2}$ surfaces. Chem. Rev. 113, 3887-3948 (2013).

66. Kresse, G. \& Furthmüller, J. Efficient iterative schemes for $a b$ initio total-energy calculations using a plane-wave basis set. Phys. Rev. B 54, 169-186 (1996).

67. Krukau, A. V., Vydrov, O. A., Izmaylov, A. F. \& Scuseria, G. E. Influence of the exchange screening parameter on the performance of screened hybrid functionals. J. Chem. Phys. 125, 224106 (2006).

68. Blöchl, P. E. Projector augmented-wave method. Phys. Rev. B 50, 17953-17979 (1994).

69. Monkhorst, H. J. \& Pack, J. D. Special points for Brillouin-zone integrations. Phys. Rev. B 13, 5188-5192 (1976).

\section{Acknowledgements}

We acknowledge the National Science Foundation of China (21006068, 21222604 and U1463205), Specialized Research Fund for the Doctoral Program of Higher Education (20120032110024), the Scientific Research Foundation for the Returned Overseas Chinese Scholars (MoE), the Ministry of Education of China (IRT-13R30 and IRT-13022) and the Program of Introducing Talents of Discipline to Universities (B06006 and B12015) for financial support.

\section{Author contributions}

J.G., L.L. and J.Y. conceived and designed the experiments, analysed the results and wrote the manuscript. J.G., L.L., J.Y. and T.W. performed the experiments and analysed the results. Z.Z. and J.G. carried out DFT calculations and analysed results. All authors contributed to the discussions of the results in this manuscript.

\section{Additional information}

Supplementary Information accompanies this paper at http://www.nature.com/ naturecommunications

Competing financial interests: The authors declare no competing financial interests.

Reprints and permission information is available online at http://npg.nature.com/ reprintsandpermissions/

How to cite this article: Li, L. et al. Sub-10 nm rutile titanium dioxide nanoparticles for efficient visible-light-driven photocatalytic hydrogen production. Nat. Commun. 6:5881 doi: 10.1038/ncomms6881 (2015) 